\title{
Relação entre o desenvolvimento infantil e as fissuras labiopalatinas
}

\author{
Relationship between child development \\ and cleft lip and palate
}

\author{
Relación entre desarrollo infantil \\ y labio leporino y paladar hendido
}

\author{
Marina Silveira Schönardie* ii \\ Leticia Pacheco Ribas* \\ Gabriela Peretti Wagner* (i) \\ Maria Cristina de Almeida Freitas Cardoso*
}

\section{Resumo}

Introdução: As fissuras labiopalatinas são malformações congênitas recorrentes e ocasionam prejuízos de fala, deglutição, audição e socialização. O desenvolvimento infantil compreende a evolução das habilidades motoras, cognitivas, sociais e emocionais, sendo influenciado por muitos fatores. Objetivo: investigar o desenvolvimento infantil de crianças com fissuras labiopalatinas (FLP). Método: Estudo observacional transversal realizado por análise de dados de entrevista e do resultado do teste de Denver II em um grupo de crianças com FLP, sem síndromes associadas. Foram incluídos 27 indivíduos entre sete e 68 meses, de ambos os sexos, que realizam acompanhamento fonoaudiológico em ambulatório do Sistema Único de Saúde de um hospital pediátrico. Resultados: A mediana de idade dos 27 participantes foi 25 meses, sendo 15 indivíduos do sexo feminino $(55,6 \%) ; 13$ com fissura pós-forame incisivo incompleta (48,1\%); a maioria, o primogênito da família (51,85\%); não tendo iniciado atividades escolares $(59,26 \%)$. A média de idade materna foi quatro anos mais baixa que a paterna e a renda familiar é de cerca de dois salários mínimos. A FLP transforame unilateral ocorreu mais em meninos $(55,6 \%)$, enquanto a fissura palatina pós-forame incompleta ocorreu mais em meninas (61,5\%). No teste aplicado, a área da linguagem foi a mais acometida, tendo $37 \%$ dos indivíduos apresentando item de cautela ou atenção, com falha em uma prova. Conclusão: Observou-se desenvolvimento esperado na maioria da população estudada, possivelmente ocasionado pelo atendimento precoce recebido. Os resultados com risco para

\footnotetext{
* Hospital da Criança Santo Antônio - Irmandade Santa Casa de Misericórdia de Porto Alegre - Porto Alegre, RS, Brasil.

Contribuição dos autores:

MSS: realizou o delineamento do estudo, a coleta, a análise e a interpretação dos dados; redigiu o artigo inicial. LPR e GPW: realizaram a análise e a interpretação dos dados do artigo.

MCAFC: concebeu e delineou o estudo, realizou a análise e interpretação dos dados e aprovou a versão final a ser publicada.

E-mail para correspondência: Marina Silveira Schönardie - marinaschonardie@hotmail.com Recebido: 06/05/2020

Aprovado: 20/10/2020
} 
atraso ocorreram mais frequentemente no sexo feminino, nas fissuras pós-forame, no primogênito e relacionaram-se com a área de linguagem.

Palavras-chave: Desenvolvimento Infantil; Anormalidades Congênitas; Linguagem Infantil; Fenda Labial; Fissura Palatina.

\section{Abstract}

Introduction: Lip-palatine clefts are recurrent congenital malformations and cause speech, swallowing, hearing and socialization impairment. Child development comprises the evolution of motor, cognitive, social and emotional skills and is influenced by many factors. Objective: To investigate the child development of subjects with cleft lip and palate (CLP). Method: Cross-sectional observational study conducted by interview and Denver II assessment in a group of children with CLP without associated syndromes. We included 27 individuals between seven and 68 months, of both sexes, who perform speech therapy follow-up in the Unified Health System outpatient clinic in a pediatric hospital. Results: The median age of the 27 participants was 25 months, 15 female (55.6\%). 13 participants presented incomplete incisive post-foramen cleft (48.1\%) and most are the first child in the family $(51.85 \%)$, having not started school activities (59.26\%). The average maternal age was 4 years lower than the paternal and the family income is about two minimum wages. One-sided transforamen cleft occurred more in boys $(55.6 \%)$, while post-incomplete foramen cleft palate occurred more in girls $(61.5 \%)$. In the applied test, the language area was the most affected, with $37 \%$ of individuals presenting item of caution or attention, with failure in a test. Conclusion: Normal development was observed in most of the studied population, possibly caused by the early care received. Results at risk for delay occurred more frequently in females, in the post-foramen clefts, in the firstborn and were related to the language area.

Keywords: Child Development; Congenital Abnormalities; Child Language; Cleft Lip; Cleft Palate.

\section{Resumen}

Introducción: Las fisuras del paladar y labio leporino son malformaciones congénitas recurrentes. Causan alteraciones del habla, deglución, audición y socialización. El desarrollo infantil comprende la evolución de las habilidades motoras, cognitivas, sociales, emocionales y está influenciado por muchos factores. Objetivo: investigar el desarrollo infantil de niños con labio leporino y paladar hendido (CLP). Método: estudio observacional transversal realizado por entrevista y análisis de resultados de Denver II en un grupo de niños con fisura del paladar sin síndromes asociados. Se incluyeron 27 individuos entre siete y 68 meses, ambos sexos, que realizan seguimiento de terapia del habla en la clínica ambulatoria del Sistema Único de Salud en un hospital pediátrico. Resultados: La mediana de edad de los 27 participantes fue de 25 meses, 15 mujeres $(55,6 \%) .13$ presentaron hendidura incisiva post-foramen incompleta $(48.1 \%)$. La mayoría son el primer hijo (51.85\%), que no han comenzado las actividades escolares (59.26\%). La edad materna promedio fue 4 años menor que la paterna y el ingreso familiar es de aproximadamente dos salarios mínimos. La fisura transforamen unilateral ocurrió más en niños (55.6\%), mientras que el paladar hendido post-incompleto ocurrió más en niñas (61.5\%). En la prueba aplicada, el área de lenguaje fue la más afectada, con el 37\% de las personas presentando un elemento de precaución o atención, con fracaso en una prueba. Conclusión: Se observó desarrollo normal en la mayoría de la población estudiada, posiblemente debido a la atención temprana recibida. Los resultados con riesgo de retraso ocurrieron con mayor frecuencia en las mujeres, en las hendiduras posteriores al foramen, en el primogénito y se relacionaron con área de lenguaje.

Palabras clave: Desarrollo Infantil; Anomalías Congénitas; Lenguaje Infantil; Fisura del Paladar; Labio Leporino. 


\section{Introdução}

As fissuras lábio-palatinas (FLP) são malformações congênitas de origem multifatorial que envolvem condições genéticas e ambientais. A fenda labial e/ou palatina é a anomalia congênita mais comum na face ${ }^{1}$ e ocorre devido ao não fusionamento dos processos craniofaciais embrionários que originam palato primário e secundário, que deveria acontecer entre a quarta e a décima segunda semana de gestação².

Segundo a Organização Mundial da Saúde $(\mathrm{OMS})^{3}$, a incidência média de FLP transforame incisivas na população ocidental é de 1:1000 nascimentos, enquanto que as fissuras somente de palato têm incidência de 1:2000 nascimentos. Cabe ressaltar que esses índices variam de acordo com fatores étnicos e geográficos, sendo que a variação de etnia mostra-se uma característica mais importante do que a geográfica para a incidência das fissuras ${ }^{3}$.

A incidência de FLP no Brasil varia entre $0,47 \mathrm{e}$ 1,54 por 1000 nascidos e de etiologia multifatorial, associada ou não aos fatores de hereditariedade e aos aspectos clínicos maternos como estresse, infecções, medicamentos e/ou irradiações ${ }^{4,5}$.

Os achados epidemiológicos apontam que os fatores econômicos municipais, com base no censo realizado em 2000, não estão relacionados com a prevalência de fendas orofaciais na população. Contudo, podera-se que esse resultado pode ter sido influenciado pelas falhas de registro, subestimando o número de indivíduos com a malformação em municípios menos desenvolvidos ${ }^{6}$.

A classificação das FLP envolve características anatômicas e a região acometida. Considera-se o forame incisivo como referência morfológica e embrionária, podendo ser classificadas em pré, pós ou transforme incisivo. Podem ser, ainda, unilaterais ou bilaterais e completas ou incompletas ${ }^{7}$. As FLP comprometem, em graus variados, a linguagem oral, a fala e a audição, podendo gerar prejuízos nas interações sociais ${ }^{8}$.

O desenvolvimento infantil, assim como o crescimento, é influenciado por fatores geneticamente determinados e por fatores ambientais, como o meio social em que a criança está inserida9. Dessa forma, o desenvolvimento deve ser analisado através de uma avaliação que contemple funções cognitivas complexas da criança9.

Enquanto o crescimento relaciona-se com aspectos físicos, como peso corporal e estatura, o desenvolvimento compreende, além dos aspectos fisiológicos, o processo de maturação dos órgãos e sistemas. Sendo assim, o desenvolvimento infantil possibilita maior eficiência na realização das funções e na aquisição de habilidades cada vez mais elaboradas com o decorrer do tempo ${ }^{9}$.

No início da vida, o bebê percebe as sensações e as replica através de ações, sendo a exploração do ambiente um aspecto fundamental para o desenvolvimento do seu esquema corporal. A percepção e a cognição se desenvolvem e têm sua expressão através da atividade motora. Além dos aspectos sensoriais e motores, existe, ainda, o fator afetivo, elemento básico para motivar as ações. O resultado da interação desses fatores define como ocorre o processo de desenvolvimento de cada criança, a partir das suas possibilidades e do ambiente em que está inserida ${ }^{10}$.

A interação social é fundamental para o desenvolvimento infantil e pode prejudicar as relações sociais das crianças com FLP, gerada pela escassez de estímulos adequados ${ }^{11}$. Essa carência pode estar associada aos déficits de linguagem e de audição ${ }^{12}$, que interferem no processo do desenvolvimento neuropsicológico ${ }^{13}$, visto que há predisposição para perda auditiva nas FLP, podendo ser indicador de risco para o desenvolvimento de competências linguísticas ${ }^{14}$. Há, portanto, possibilidade de relação entre a alteração no desenvolvimento infantil, as habilidades de linguagem e a presença de FLP ${ }^{8}$.

Achados de uma metanálise, realizada com crianças pequenas com FLP, sugerem que nessa população o processo de construção de fala e linguagem sofre atraso, quando comparado com o processo de crianças na mesma faixa etária, porém sem a malformação. Observou-se também que esse atraso tende a diminuir com o aumento da idade ${ }^{15}$.

Vários fatores podem desencadear algum tipo de alteração no desenvolvimento infantil, havendo indícios recentes na literatura que apontam que as FLP podem comprometer aspectos neuropsicológicos, além de acometer áreas já sabidas como a fala, a linguagem e a audição ${ }^{8,13}$. Entretanto, há estudos em que não se encontra associação entre o risco para alteração de linguagem ou diferenças significativas no desempenho dessa habilidade em crianças com malformação ${ }^{16}$.

Há diferentes protocolos descritos na literatura dedicados a avaliar o desenvolvimento infantil, dentre eles o Teste de Denver II, que realiza uma triagem do desenvolvimento. Tal instrumento pode 
ser aplicado em crianças de zero a seis anos e possui 125 itens que buscam avaliar quatro grandes áreas: pessoal-social, motor fino-adaptativo, linguagem e motor grosso. A sua interpretação se divide em normal, questionável/risco para o desenvolvimento e não aplicável, e depende dos itens realizados ou não pela criança, de acordo com a sua idade ${ }^{17}$. Possui aplicação rápida, levando em torno de 30 minutos e é recomendado pela Sociedade Brasileira de Pediatria e pela Academia Americana de Pedia$\operatorname{tria}^{18}$. O teste de Denver II foi traduzido e adaptado transculturalmente para ser utilizado no Brasil ${ }^{19}$.

Vista a possibilidade de ocorrência de alterações do desenvolvimento relacionadas às crianças com FLP e a necessidade de acompanhamento precoce dessas crianças, a presente pesquisa teve como objetivo investigar o desenvolvimento infantil de crianças com fissuras labiopalatinas, através da aplicação do Teste de Denver II.

\section{Método}

Trata-se de um estudo observacional e transversal, de caráter quantitativo e exploratório, que investigou a população de crianças com FLP atendidas por um projeto de extensão de uma universidade federal, que acontece semanalmente nos ambulatórios de especialidades do Sistema Único de Saúde - SUS, em um hospital pediátrico de Porto Alegre/RS.

Este estudo foi aprovado pelo Comitê de Ética em Pesquisa da instituição de saúde, em coparticipação com a Universidade sob o parecer número 3.398.845. Todos os responsáveis pelas crianças participantes do estudo assinaram o Termo de Consentimento Livre e Esclarecido (TCLE).

Os participantes deste estudo fazem parte do projeto de extensão "Fissuras Lábio-Palatinas", que conta com um banco de dados composto por contato telefônico, identificação e tipo de fissura de 68 crianças em atendimento clínico ou em gerenciamento no período de 2015 a 2019. As crianças com FLP são encaminhadas para o grupo de atendimento fonoaudiológico através de interconsultas dos profissionais que compõem o corpo clínico dos ambulatórios de especialidades do SUS da instituição de saúde. Em geral, as interconsultas são solicitadas pelos profissionais das áreas de cirurgia plástica, otorrinolaringologia pediátrica ou cirurgia bucomaxilofacial.
Foram incluídos na amostra dados de indivíduos de ambos os sexos, com idade entre sete e 68 meses, diagnosticados com a malformação e que fazem parte deste projeto. Foram excluídos aqueles com síndromes genéticas e que não compareceram ou não concordaram em participar deste estudo.

O protocolo desta pesquisa foi executado entre os meses de junho e agosto de 2019 e contou com a realização dos seguintes procedimentos:

- Contato pessoal ou por telefone com os responsáveis pelas crianças listadas no banco de dados do projeto de extensão universitária, apresentação desse projeto de pesquisa, anuência e assinatura dos TCLE;

- Breve entrevista inicial com os dados de identificação pessoal dos participantes: nome, sexo, data de nascimento, idade materna e paterna ao nascimento, ordem de nascimento do indivíduo da pesquisa, características da FLP (áreas acometidas e sua extensão), escolaridade da criança, renda familiar e revisão do telefone para contato;

- Aplicação do Teste de Denver II $^{17}$ realizado pela pesquisadora assistente, sendo verificados os itens de intersecção pela linha da idade do participante. $\mathrm{O}$ teste é um instrumento de triagem das condições de desenvolvimento da criança com 125 itens, distribuídos em quatro áreas/categorias: motor-grosseiro, motor fino-adaptativo, linguagem e pessoal-social. A sua interpretação considera o desenvolvimento como "normal" quando observado nenhum ou um único item "cautela ou risco" e "questionável" quando apresentados dois ou mais itens cautela. Considera-se como "cautela ou risco", aquelas áreas em que o indivíduo falhou em uma prova (ou item), no qual mais de $75 \%$ a $90 \%$ das crianças já realizam, como preconiza o teste. Estes resultados foram digitados nos prontuários eletrônicos de atendimentos de cada participante, que segue em gerenciamento ou atendimento clínico fonoaudiológico no mesmo local;

- Os dados foram computados em uma tabela Excel ${ }^{\circledR}$ para posterior análise estatística.

Para a análise das variáveis quantitativas foram estabelecidas média, mediana e desvio padrão, e para variáveis qualitativas (tipo de fissura, sexo, interpretação do teste, áreas específicas analisados no teste) foram estabelecidas as frequências absolutas e relativas. A associação entre as variáveis averiguadas foi verificada através do Teste de Qui-Quadrado ou do teste Mann-Whitney, dependendo 
do número absoluto da variável. Para análise dos dados foi utilizado o Statistical Package for the Social Sciences (SPSS) versão 23.0 para Windows. O nível de significância adotado para os testes estatísticos foi de $5 \%$.

\section{Resultado}

Dos 68 indivíduos atendidos no período de vigência do projeto de extensão, 33 com idade superior aos seis anos foram excluídos. Dos 35 indivíduos contatados com idade até seis anos, foram excluídos três com quem não foi possível realizar contato telefônico; dois que apresentaram síndromes genéticas, devido à possibilidade de influência sobre os resultados do teste; dois que negaram o atendimento quando contatados e um devido a óbito, totalizando uma amostra de 27 participantes. Dos participantes incluídos na amostra, 25 estavam em acompanhamento fonoaudiológico no ambulatório de especialidades do SUS da instituição de saúde proponente deste estudo, um estava inserido nesse atendimento na cidade onde reside e, outro havia recebido alta do atendimento.

A idade mínima dos participantes foi sete meses, idade máxima 68 meses, com uma mediana de idade de 25 meses. O sexo de maior incidência foi o feminino $(55,6 \% ; n=15)$ e o tipo de fissura mais incidente foi a pós-forame incisivo incompleta $(48,1 \% ; n=13)$. A caracterização da amostra se encontra na Tabela 1.

Tabela 1. Caracterização da amostra

\begin{tabular}{|c|c|c|c|}
\hline Variáveis & & n (\%) & $\mathrm{md} / \mathrm{m}$ \\
\hline Idade & $\begin{array}{l}\text { Mínima } \\
\text { Máxima }\end{array}$ & $\begin{array}{c}7 \text { meses } \\
68 \text { meses }\end{array}$ & $\mathrm{md}=25$ meses \\
\hline Sexo & $\begin{array}{l}\text { Masculino } \\
\text { Feminino }\end{array}$ & $\begin{array}{l}12(44,4) \\
15(55,6)\end{array}$ & \\
\hline Tipo de fissura & $\begin{array}{c}\text { Transforame Unilateral } \\
\text { Transforame bilateral } \\
\text { Pós-forame incompleta } \\
\text { Pré-forame }\end{array}$ & $\begin{array}{c}9(33,3) \\
2(7,4) \\
13(48,1) \\
3(11,2)\end{array}$ & \\
\hline
\end{tabular}

Legenda: $\mathrm{n}=$ número de sujeitos; \%= porcentagem; $\mathrm{md}=$ mediana

Quanto à ordem de nascimento, $51,85 \%(\mathrm{n}=14)$ dos participantes da pesquisa são o primeiro filho; $25,92 \%(n=7)$ são o segundo; $14,81 \%(n=4)$ são o terceiro; $3,70 \%$ (n=1) são o quarto e outros $3,70 \%$ $(\mathrm{n}=1)$ são o quinto filho. A idade materna no nascimento teve uma média de 26,85 anos e a paterna de 31,22 anos. As crianças, em sua maioria (59,26\%; $\mathrm{n}=16$ ), não iniciaram atividades escolares e, entre as que iniciaram $(40,74 \% ; n=11)$, o sexo feminino teve maior incidência $(63,63 \% \mathrm{n}=7)$. A média de idade das crianças que iniciaram na escola foi de 35 meses, sendo que a média de idade de inserção na escola das meninas foi 33,57 meses, e dos me- ninos de 37,5 meses. A média da renda familiar das crianças avaliadas é de aproximadamente dois salários mínimos ( $\mathrm{R} \$ 1951,85)$.

As fissuras palatinas pós-forame incompleta tiveram maior incidência nas meninas $(61,5 \%$ $\mathrm{n}=8$ ) e as FLP transforame unilateral tiveram maior incidência em meninos $(55,6 \% n=5)$. As FLP transforame bilateral e as fissuras pré-forame unilateral foram equivalentes entre meninos e meninas. Não houve diferença estatística na associação entre o tipo de fissura e o sexo $(\mathrm{p}=0,82)$. Os dados comparativos encontram-se na Tabela 2. 
Tabela 2. Tipo de fissura e sexo

\begin{tabular}{lcccc}
\hline \multirow{2}{*}{ Tipo de Fissura } & \multicolumn{2}{c}{ Sexo } & Total & \multirow{2}{*}{ p* } \\
\cline { 2 - 3 } & $\mathbf{M}$ & $\mathbf{n}(\%)$ & $\mathbf{n}(\%)$ & \\
\hline FLP transforame unilateral & $5(55,6)$ & $4(44,4)$ & $9(100)$ & \\
FLP transforame bilateral & $1(50,0)$ & $1(50,0)$ & $2(100)$ & 0,82 \\
Fissura palatina pós-forame incompleta & $5(38,5)$ & $8(61,5)$ & $13(100)$ & \\
Fissura pré-forame unilateral & $1(33,3)$ & $2(66,7)$ & $3(100)$ & \\
Total & $12(44,4)$ & $15(55,6)$ & $27(100)$ & \\
\hline
\end{tabular}

Legenda: $F L P=$ fissura labiopalatina; $M=$ masculino; $F=$ feminino; $n=$ número absoluto; $\%=$ número relativo; $\mathrm{p}^{*}=$ teste $\mathrm{Qui}$-quadrado

Quanto à interpretação dos resultados do Teste de Denver II, 18 crianças apresentaram resultado normal $(66,7 \%)$, enquanto nove apresentaram resultado questionável $(33,3 \%)$, cuja associação com o sexo não evidenciou diferença estatística $(\mathrm{p}=0,68)$. Os dados detalhados da associação entre o resultado do teste Denver II ao sexo encontram-se na Tabela 3.

Tabela 3. Associação entre o resultado do Teste de Denver II e o sexo

\begin{tabular}{lcccc}
\hline $\begin{array}{l}\text { Resultado do Teste } \\
\text { de Denver II }\end{array}$ & $\begin{array}{c}\text { Meninas } \\
\mathbf{n}(\%)\end{array}$ & $\begin{array}{c}\text { Meninos } \\
\mathbf{n}(\%)\end{array}$ & $\begin{array}{c}\text { Total } \\
\mathbf{n}(\%)\end{array}$ & p* \\
\hline Normal & $9(60)$ & $9(75)$ & $18(66,7)$ & \\
Questionável & $6(40)$ & $3(25)$ & $9(33,3)$ & 0,68 \\
Total n (\%) & $15(100)$ & $12(100)$ & $27(100)$ & \\
\hline
\end{tabular}

Legenda: $\mathrm{n}=$ número absoluto; $\%=$ número relativo; $\mathrm{p}^{*}=$ teste de Fisher

A associação entre o resultado do teste de Denver II e a idade dos participantes desta pesquisa se mostrou independente frente à idade da criança (para os resultados normais de 24 meses e para os questionáveis de 25 meses), não havendo associação significativa entre essas duas variáveis, verificada através do teste Mann-Whitney $(\mathrm{p}=1,0)$.

Foram encontrados itens indicativos de "cautela ou risco" nas quatro áreas investigadas pelo
Teste de Denver II. Na área que avalia a linguagem, 10 participantes apresentaram itens indicativos de cautela (37\%); na área correspondente a motor fino-adaptativo, seis $(22,2 \%)$; no campo pessoal-social, quatro (14,8\%); e, na avaliação da área motor grosso, quatro crianças $(14,8 \%)$. Os dados comparativos e a associação entre os resultados do teste de Denver II e o sexo encontram-se na Tabela 4.

Tabela 4. Associação entre as áreas analisadas pelo Teste de Denver II, o sexo e o número de "cautela"

\begin{tabular}{|c|c|c|c|c|c|c|c|}
\hline \multirow{2}{*}{$\begin{array}{l}\text { Áreas do Teste } \\
\text { Denver II }\end{array}$} & \multirow[b]{2}{*}{ Sexo } & \multicolumn{5}{|c|}{ Número de Cautela n (\%) } & \multirow[b]{2}{*}{$\mathbf{p}$} \\
\hline & & $1(\%)$ & $2(\%)$ & $3(\%)$ & $4(\%)$ & $\begin{array}{l}\text { Total } \\
\text { n (\%) }\end{array}$ & \\
\hline Linguagem & $\begin{array}{c}\text { meninas } \\
\text { meninos } \\
\text { geral }\end{array}$ & $\begin{array}{c}4(57,1) \\
1(33,3) \\
5(50)\end{array}$ & $\begin{array}{c}2(28,6) \\
0(0) \\
2(20)\end{array}$ & $\begin{array}{c}1(14,3) \\
1(33,3) \\
2(20)\end{array}$ & $\begin{array}{c}0(0) \\
1(33,4) \\
1(10)\end{array}$ & $\begin{array}{c}7(100) \\
3(100) \\
10(100)\end{array}$ & $0,28^{*}$ \\
\hline Motor fino-adaptativo & $\begin{array}{c}\text { meninas } \\
\text { meninos } \\
\text { geral }\end{array}$ & $\begin{array}{l}3(100) \\
3(100) \\
6(100)\end{array}$ & $\begin{array}{l}0(0) \\
0(0) \\
0(0)\end{array}$ & $\begin{array}{l}0(0) \\
0(0) \\
0(0)\end{array}$ & $\begin{array}{l}0(0) \\
0(0) \\
0(0)\end{array}$ & $\begin{array}{l}3(100) \\
3(100) \\
6(100)\end{array}$ & $* *$ \\
\hline Pessoal-social & $\begin{array}{c}\text { meninas } \\
\text { meninos } \\
\text { geral }\end{array}$ & $\begin{array}{c}3(100) \\
0(0) \\
3(75)\end{array}$ & $\begin{array}{l}0(0) \\
0(0) \\
0(0)\end{array}$ & $\begin{array}{c}0(0) \\
1(100) \\
1(25)\end{array}$ & $\begin{array}{l}0(0) \\
0(0) \\
0(0)\end{array}$ & $\begin{array}{l}3(100) \\
1(100) \\
4(100)\end{array}$ & $0,25 * * *$ \\
\hline Motor grosso & $\begin{array}{c}\text { meninas } \\
\text { meninos } \\
\text { geral }\end{array}$ & $\begin{array}{c}2(100) \\
0(0) \\
2(50)\end{array}$ & $\begin{array}{c}0(0) \\
2(100) \\
2(50)\end{array}$ & $\begin{array}{l}0(0) \\
0(0) \\
0(0)\end{array}$ & $\begin{array}{l}0(0) \\
0(0) \\
0(0)\end{array}$ & $\begin{array}{l}2(100) \\
2(100) \\
4(100)\end{array}$ & $0,33 * * *$ \\
\hline
\end{tabular}

Legenda: $\mathrm{n}=$ número absoluto; $\%=$ número relativo; $\mathrm{p}^{*}=$ teste Qui-quadrado; $\mathrm{p}^{* *}=$ constante; $\mathrm{p}^{* * *}=$ teste de Fisher; $1=$ uma falha; $2=$ duas falhas; $3=$ três falhas; $4=$ quatro falhas. 


\section{Discussão}

As FLP são malformações que necessitam de atendimento multiprofissional desde o nascimento, devido ao comprometimento das estruturas faciais que acarretam disfunções orais, podendo desencadear alterações no desenvolvimento global da criança e interferir na qualidade de vida ${ }^{8,13}$.

A influência da ordem de nascimento com a ocorrência das FLP tem sido estudada. Uma metanálise realizada nos Estados Unidos revelou associação positiva entre as variáveis, cujos resultados apontam para o aumento da ocorrência de casos de FLP na família, vinculado ao aumento na ordem de nascimento dos filhos ${ }^{20}$. Na presente pesquisa, os indivíduos acometidos pela malformação são, em maior número, o primeiro ou o segundo filho, de acordo com esta metanálise ${ }^{20}$ e por outra pesquisa, na qual foi verificada uma proporção semelhante à do presente estudo, em que $74 \%$ da amostra estudada nasceu na primeira ou na segunda gestação materna ${ }^{21}$.

Em referência à idade dos pais em relação ao nascimento da criança com FLP, encontram-se dados de idade média paterna de 29,5 anos e materna de 25,7 anos, além de aumento da incidência de FLP com o aumento da idade dos pais ${ }^{22}$. Alguns estudos apontam a idade paterna mais elevada em relação à idade materna dos indivíduos com FLP22. Este estudo encontrou uma média de idade próxima ao encontrado na literatura, e uma tendência da idade paterna superior em 4 anos à idade materna, também em conformidade com a literatura.

Outro fator predisponente das FLP é a renda familiar. No Brasil, a estratificação das classes socioeconômicas tem como referência a renda média domiciliar, sendo divididas em A, B1, B2, C1, C2 e D-E, da mais alta para a mais baixa. No presente estudo, a renda familiar média corresponde à inclusão dos indivíduos no estrato sócio econômico C2 (renda média), o que não corresponde aos achados de outros artigos, que verificaram maior incidência das FLP em indivíduos de mais baixa renda ${ }^{2}$.

A renda socioeconômica é considerada, também, como um dos fatores para o comprometimento do desenvolvimento infanti ${ }^{23}$. Resultados de uma pesquisa sobre o desenvolvimento que avaliou crianças brasileiras da região sul do país a partir da influência de variáveis ambientais, utilizando o Teste de Denver II, mostra a relação entre baixa renda e baixa escolaridade com o comprometimento do desenvolvimento da linguagem, devido à privação de estímulo e às situações de estresse vividas ${ }^{24}$.

Os comprometimentos da linguagem em crianças com essa malformação são associados à falta de estimulação adequada e às suas alterações de fala, que acometem, principalmente, a ressonância vocal e a articulação das palavras, envolvendo tanto os aspectos fonéticos quanto os fonológicos ${ }^{11}$.

A ocorrência de FLP é mais frequente nos homens, enquanto que as fissuras isoladas de palato acometem mais mulheres ${ }^{1,3}$. O presente estudo evidencia a prevalência maior do sexo feminino e da fissura do tipo pós-forame incisivo incompleta, dados que estão de acordo com o encontrado na literatura, visto que está descrita maior incidência das fissuras isoladas de palato no sexo feminino e das FLP no sexo masculino.

O termo desenvolvimento encontrado na literatura varia conforme os autores, sendo referido como infantil, neuropsicológico e/ou neuropsicomotor $^{23-25}$. O desenvolvimento infantil apresenta pontos críticos que devem ser observados. Alguns deles são mais vulneráveis aos fatores ambientais, podendo levar a transtornos do desenvolvimento devido à privação de estímulos ${ }^{25}$. $\mathrm{O}$ termo desenvolvimento neuropsicológico (DNP) envolve diferentes domínios (linguagem, motor, social, entre outros). A expressão DNP não privilegia uma faceta em detrimento da outra, não separa os aspectos neurológicos dos motores, referindo-se, também, aos processos perceptivos e às habilidades cognitivas complexas como as sociais, motivacionais e de linguagem, contemplando o aspecto sensitivo-sensorial ${ }^{25}$. Acredita-se que DNP seja uma expressão que mais de acordo com o estudo realizado, devido às diferentes áreas avaliadas pelo Teste de Denver.

Quanto à associação das FLP com alterações no DNP, alguns estudos encontraram uma relação associativa entre as variáveis ${ }^{8,26,27}$. Este estudo encontrou uma incidência maior de resultados pontuados como normais no Teste de Denver II, diferente do encontrado nos estudos de Cavalheiro ${ }^{8}$ e Jurado ${ }^{27}$. Os resultados questionáveis ocorreram no sexo feminino em maior número. Na literatura, não foram encontrados dados referentes ao sexo utilizando o mesmo teste na mesma população, porém, em indivíduos sem FLP, os achados da literatura divergem dos encontrados neste estudo, pois os dados sugerem o predomínio do sexo masculino em casos de alterações no processo de DNP ${ }^{28}$. 
Quanto ao número de filhos e a sua ordem de nascimento relacionada às alterações do desenvolvimento infantil, encontra-se na literatura que crianças fissuradas com mais de três irmãos apresentam maior probabilidade de possuir alterações no desenvolvimento ${ }^{23}$. Esta associação pode ser verificada junto aos participantes desta pesquisa, visto que as crianças avaliadas apresentaram, em maior número, resultado normal no DNP e, a maioria delas, tem nenhum ou um único irmão.

$\mathrm{Na}$ associação da renda familiar com o DNP verificou-se, junto aos dados dos participantes deste estudo, não ser fator de risco para DNP questionável, pois a maior parte dos indivíduos obteve resultado normal no Teste de Denver II, diferente do encontrado na literatura ${ }^{23}$.

Na realização do Teste de Denver II, a área avaliada com maior incidência de indivíduos com itens cautela, indicando pior desempenho da função, foi da linguagem, seguida da área motor fino-adaptativo, pessoal social e motor grosso, esses dois últimos equiparados. Dados semelhantes, indicando maior alteração na área de linguagem, foram encontrados em um estudo realizado no estado de São Paulo (BR), que avaliou o DNP de crianças com FLP através do mesmo teste ${ }^{8}$.

$\mathrm{O}$ uso do Teste de Denver junto à população estudada mostrou-se adequado, de rápida aplicação e de fácil interpretação. Quanto à relevância de sua aplicação, acredita-se que, através dos resultados obtidos e dos marcos do desenvolvimento estabelecidos pelo instrumento, podem ser realizadas intervenções terapêuticas mais eficientes. Considera-se importante identificar precocemente alterações relacionadas ao processo do desenvolvimento infantil, pois a partir da detecção do risco de atraso, podem ser estabelecidas estratégias de intervenção desde os primeiros anos de vida, prevenindo possíveis distúrbios ${ }^{29}$.

Quanto ao atendimento fonoaudiológico precoce específico de crianças com FLP, tem-se que esse atendimento é necessário desde o período neonatal, passando pelo pré-cirúrgico e seguindo após as cirurgias corretivas de lábio e palato. $\mathrm{O}$ atendimento precoce deve ser realizado com orientações aos familiares em relação ao tratamento, sobre aspectos de higiene bucal, massagens, alimentação e estimulação de fala e linguagem, auxiliando na compreensão da malformação ${ }^{30}$.

Identificar e tratar precocemente problemas de linguagem nessa população é tido como impor- tante, pois pode evitar dificuldades no processo de aprendizagem e de socialização, comprometendo o DNP ${ }^{8}$. Este estudo, no entanto, encontrou DNP normal na avaliação do desenvolvimento aplicada com essa população, o que poderia ser explicado pelo acompanhamento periódico e especializado estendido aos participantes desde os primeiros dias de vida, ocorrendo com frequência semanal, quinzenal ou mensal, no qual se orientam e realizam-se estimulações adequadas.

\section{Conclusão}

As variáveis verificadas evidenciaram desenvolvimento normal em maior incidência junto à população estudada, o que pode ser, possivelmente, justificado pelo atendimento fonoaudiológico especializado dispensado precocemente e de forma periódica a este grupo de crianças. Os atendimentos oferecidos envolvem estímulos perceptivos e de habilidades cognitivas complexas, orientações e intervenções pontuais que auxiliam o processo do DNP.

Os resultados questionáveis, ou seja, com risco para o desenvolvimento, se apresentaram no sexo feminino, nas fissuras pós-forame e em indivíduos que eram o primeiro filho, acometendo, mais frequentemente, a área de linguagem.

O estudo possui limitações quanto ao tamanho da amostra e o tempo reduzido para a realização da coleta, sendo, portanto, sugeridos novos estudos com um maior número de participantes para que os resultados possam ser generalizados. Além disso, a pesquisa utilizou somente o Teste de Denver II para a avaliação do DNP, sendo necessária a realização de novos estudos com crianças com FLP utilizando outros instrumentos/testes, para uma avaliação mais completa e ampla do desenvolvimento dessa população.

\section{Referências bibliográficas}

\footnotetext{
1. Martelli DBR, Machado RA, Swerts MSO, Rodrigues LAM, Aquino SND, Martelli Júnior H. Non syndromic cleft lip and palate: relationship between sex and clinical extension. Braz J Otorhinolaryngol. 2012; 78(5): 116-20.
}

2. Kuhn VD, Miranda C, Dalpian DM, de Moraes CMB, Backes DS, Martins JS, dos Santos BZ. Fissuras labiopalatais: revisão da literatura. Disciplinarum Scientia| Saúde. 2016; 13(2): 237-45. 
3. WHO: World Health Organization. Global strategies to reduce the health-care burden of craniofacial anomalies: Report of WHO meetings on international collaborative research on craniofacial anomalies. [cited 2002]. Disponível em: http:// www.who.int/ncd/hgn/publications. Acesso em: 04 de maio de 2020 .

4. Luiza A, Góis DN, Santos JASS, Oliveira RLB, Silva LCF. A Descriptive Epidemiology Study of Oral Cleft in Sergipe, Brazil. Int Arch Otorhinolaryngol. 2013; 17: 390-4.

5. Nunes LMN, Queluz DP, Pereira AC. Prevalência de fissuras labiopalatais no município de Campos dos Goytacazes-RJ. Rev Bras Epidemiol. 2007; 10: 109-16.

6. Rodrigues K, Sena MFD, Roncalli AG, Ferreira MAF. Prevalence of orofacial clefts and social factors in Brazil. Braz. oral res. 2009; 23(1): 38-42.

7. Spina V, Psillakis JM, Lapa FS, Ferreira MC. Classificação das fissuras lábio-palatinas: sugestão de modificação. Rev Hosp Clin Fac Med São Paulo. 1972; 27(1): 5-6.

8. Cavalheiro MG. Habilidades do desenvolvimento infantil e linguagem de crianças com fissura labiopalatina. [dissertação]. São Paulo (SP): Universidade de São Paulo; 2016.

9. Monteiro FPM, Araujo TLD, Cavalcante TF, Leandro TA, Sampaio Filho SPC. Crescimento infantil: análise do conceito. Texto \& contexto enfermagem / UFSC. 2016; 25(2): e3300014. Disponível em: https://www.scielo.br/pdf/tce/v25n2/pt_01040707-tce-25-02-3300014.pdf. Acesso em: 04 de maio de 2020.

10. Lopes SMB, LOPES JMA. Follow-up do Recém-Nascido de Alto Risco. Rio de Janeiro: Medsi; 1999.

11. Frederickson MS, Chapman KL, Hardin JM. Conversational skills of children with cleft lip and palate: a replication and extension. Cleft Palate Craniofac J. 2006; 43(2): 179-88.

12. Broen PA, Devers MC, Doyle SS, Prouty JM, Moller KT. Acquisition of linguistic and cognitive skills by children with cleft palate. J Speech Lang Hear Res. 1998; 41(3): 676-87.

13. Tabaquim MLM, Joaquim RM. Avaliação neuropsicológica de crianças com fissura labiopalatina. Arch Health Invest. 2013; 2(5): 59-67.

14. Da RéAF, Ribas LP, Machado MS, Cardoso MCAF. Relação entre os achados audiológicos e de consciência fonológica em um grupo de crianças com fissura labial e/ou palatina. Distúrb Comun, São Paulo, 2020; 32(2): 196-204.

15. Lancaster HS, Lien KM, Chow JC, Frey JR, Scherer NJ, Kaiser AP. Early Speech and Language Development in Children with Nonsyndromic Cleft Lip and/or Palate: A MetaAnalysis. J Speech Lang Hear Res. 2020; 63(1): 14-31.

16. Collett BR, Leroux B, Speltz ML. Language and early reading among children with orofacial clefts. Cleft Palate Craniofac J. 2010; 47(3): 284-92.

17. Frankenburg WK, Dodds J, Archer P. Denver II: screening manual. Denver Developmental Materials. 1990.

18. Barros L, Mistro CR. Denver II: revisão sistemática. Revista ENIAC Pesquisa. 2017; 4(1): 38-47.

19. Sabatés AL. Denver II - teste de triagem do desenvolvimento - manual técnico Editora: HOGREFE, 2013.

20. Vieira AR, Orioli IM. Birth order and oral clefts: a metaanalysis. Teratology. 2002; 66(5): 209-16.
21. Martelli DRB, da Cruz KW, de Barros LM, Silveira MF, Swerts MSO, Júnior HM. Avaliação da idade materna, paterna, ordem de paridade e intervalo interpartal para fissura lábiopalatina. Braz J Otorhinolaryngol. 2010; 76(1): 107-12.

22. Baroneza JE, de Faria MJSS, Kuasne H, do Val Carneiro JL, de Oliveira JC. Dados epidemiológicos de portadores de fissuras labiopalatinas de uma instituição especializada de Londrina, Estado do Paraná. Acta scientiarum. Health sciences. 2005; 27(1): 31-5.

23. Halpern R, Giugliani ER, Victora CG, Barros FC, Horta BL. Fatores de risco para suspeita de atraso no desenvolvimento neuropsicomotor aos 12 meses de vida. Revista chilena de pediatria. 2002; 73(5): 529-39.

24. Cachapuz RF. A influência das variáveis ambientais no desenvolvimento da linguagem em uma amostra de crianças. Rev Assoc Med Rio Grande Do Sul. 2006; 50(4): 292-301.

25. Pedroso FS. Desenvolvimento neuropsicológico. In: Cardoso MCAF. Fonoaudiologia na infância. Rio de Janeiro: Revinter; 2015. p. 1-19.

26. Nopoulos P, Berg S, Canady J, Richman L, Van Demark D, Andreasen NC. Structural brain abnormalities in adult males with clefts of the lip and/or palate. Genet Med. 2002; 4(1):1-9.

27. Jurado MRB. Habilidades do desenvolvimento global e comunicativo de crianças com fissura labiopalatina [dissertação], São Paulo (SP): Universidade de São Paulo; 2018.

28. Brito CML, Vieira GO, Costa MDCO, Oliveira NFD. Desenvolvimento neuropsicomotor: o teste de Denver na triagem dos atrasos cognitivos e neuromotores de pré-escolares. Cad Saude Publica. 2011; 27: 1403-14.

29. De Moraes MW, Weber APR, Oliveira MCS. Teste de Denver II: avaliação do desenvolvimento de crianças atendidas no ambulatório do Projeto Einstein na Comunidade de Paraisópolis. Einstein (São Paulo). 2010; 8: 149-53.

30. Leite ICG, Simões AG, Clemente MCK, Martins LS, Bittar SA, Bittar CL, Mattos VS. Fonoaudiologia hospitalar. J Bras Fonoaudiol. 2003; 4(17): 1-6. 